\title{
T-duality in supersymmetric theory of disordered quantum systems
}

\author{
A. Ossipov 1 , and V. E. Kravtsov ${ }^{1,2}$ \\ ${ }^{1}$ The Abdus Salam International Centre for Theoretical Physics, P.O.B. 586, 34100 Trieste, Italy \\ ${ }^{2}$ Landau Institute for Theoretical Physics, 2 Kosygina st., 117940 Moscow, Russia
}

\begin{abstract}
A new super-symmetric representation for quantum disordered systems is derived. This representation is exact and is dual to that of the nonlinear sigma-model. The new formalism is tested by calculating the distribution of wave function amplitudes in the 1d Anderson model. The deviation from the distribution found for a thick wire is detected near the band center $E=0$.
\end{abstract}

PACS numbers: 72.15.Rn, 72.70.+m, 72.20.Ht, 73.23.-b

Introduction.- The rigorous theory of quantum disordered systems is based almost entirely on the fieldtheoretical formulation of the nonlinear sigma-model (NL $\sigma \mathrm{M})$ 1, 2]. The most successful variant of this theory, the so called supersymmetric (SUSY) sigma-model [2, 3], allows not only for a perturbative treatment of weak localization and mesoscopic phenomena in the delocalized (metallic) phase which was previously done by the impurity diagrammatic technique [4] but also applies to systems in the localized (insulator) phase provided that the localization radius is sufficiently large. A celebrated example is the problem of localization in a thick disordered wire [3, [5] which is equivalent [6] to the Gaussian ensemble of random banded matrices with a large bandwidth $b \gg 1$.

However, despite of the great success of the nonlinear SUSY sigma-model over the last two decades [3] this approach is limited. The limitation comes from the saddlepoint approximation that locks the norm of the supermatrix field $Q$ and thus leads to a geometric constraint $Q^{2}=1$. It is precisely the validity of this saddle-point approximation that requires a quantum system to be in the metallic phase (at least for a sufficiently small length scale), or the banded random matrices to have a large bandwidth.

The best known example for which the NL $\sigma \mathrm{M}$ is insufficient is the strictly one-dimensional disordered chain described (in the position representation) by the threediagonal matrix Hamiltonian $H_{i j}=J_{i} \delta_{j, i \pm 1}+\varepsilon_{i} \delta_{i j}$. The case $J_{i}=1$, and $\varepsilon_{i}$ being a Gaussian random variable with zero mean and the variance $w=\left\langle\varepsilon_{i}^{2}\right\rangle$ is known as the 1d Anderson model. Its continuous counterpart for a weak disorder $w \ll 1$ was studied in detail in the 70 -th [7, 8, 9] with the classic results on the frequency dependent conductivity [7, 10] and dc conductance distribution [8, 9]. The special case where $\varepsilon_{i}=0$ and $J_{i}$ is a Gaussian random variable has been studied in the pioneer work by Dyson 11] and attracted a lot of interest in recent years in connection with the new symmetry classes 12]. Yet another broad class of systems which cannot be studied by the NL $\sigma \mathrm{M}$ are systems described by the almost diagonal random matrix Hamiltonian [13] with the variance of the diagonal entries $\left\langle H_{i i}^{2}\right\rangle$ much larger than that for the off-diagonal ones $\left\langle\left|H_{i \neq j}\right|^{2}\right\rangle$. Some of such systems with $\left\langle\left|H_{i \neq j}\right|^{2}\right\rangle \sim|i-j|^{-2}$ attracted recently much attention in connection with the multifractality of the critical eigenstates 13, 14, 15.

The main goal of this work is to fill in the gap in our technical ability to apply the SUSY method to quantum disordered systems. Below we present a field-theoretical description that is free of of the saddle-point approximation and thus applies to all the above cases which are not amenable to the conventional NL $\sigma \mathrm{M}$. This description keeps all the Grassmann variables and thus is different from another suggestion, the super-bosonization, which was recently proposed in Ref. [16] as an exact method instead of the NL $\sigma \mathrm{M}$ but is suffering from uncertainties and incomplete definition of the target space. The method we are proposing is similar in spirit to that of Ref. 16] but has an advantage to be as close as possible to the standard SUSY NL $\sigma \mathrm{M}$. In particular, the new supermatrix field $\hat{Q}$ can be diagonalized by a pseudo-unitary transformation with separated Grassmann and commuting variables [3] similar to the one used in the NL $\sigma \mathrm{M}$. This allows to drastically simplify calculations with the final result depending on few commuting variables only, as for the conventional NL $\sigma \mathrm{M}$.

The main property of the new description is that it is dual to the old one: the coupling matrix of the new theory is just the inverse of the coupling matrix of the NL $\sigma \mathrm{M}$. However, the space of the matrices $\hat{Q}$ of the new theory (the $\hat{Q}$ target space) is different from the $Q$ target space of the NL $\sigma \mathrm{M}$. So, we are dealing not with the conventional duality but with a kind of T-duality [17] remotely resembling the situation in the string theory.

As an illustration of the new formalism at work we consider the problem of the eigenfunction statistics for a one-dimensional Anderson model. We derive the transfer matrix integral recursive equation following the same rout as the one used in the framework of the SUSY nonlinear sigma-model for quasi-1d disordered wires [3] or banded random matrices [6]. For weak disorder this integral equation reduces to the the differential equation of exactly the same form as for a thick disordered wire. This fact (noticed earlier in [3, [5]) implies that despite the absence of the diffusive regime in a strictly $1 \mathrm{~d}$ chain, the 
smooth envelope of a wavefunction at scales much larger than the wavelength and the wire cross-section has exactly the same statistics in a thick disordered wire and in a strictly $1 \mathrm{~d}$ disordered chain.

The dual representation.-For concreteness we consider the matrix Hamiltonian of the unitary class $\mathbf{H}$ which entries $H_{i j}$ are random independent Gaussian variables characterizing by the variance matrix $\mathbf{g}_{i j}=\left\langle\left|H_{i j}\right|^{2}\right\rangle$ and the mean values $\left\langle H_{i j}\right\rangle=H_{i i}^{(0)}$. The standard SUSY field-theoretical description [3] aimed to computing the retarded/advanced (R/A) Green's functions $\left[E_{ \pm}-\mathbf{H}\right]^{-1}$ begins with introducing the $\Psi$ functional $S=\sum_{i j} \bar{\Psi}_{i}\left(E_{ \pm} \delta_{i j}-H_{i j}\right) \Psi_{j}$, where $E_{ \pm}=E \pm \tilde{\omega} / 2$ and $\tilde{\omega}=\omega+2 i \eta$ with $\eta \rightarrow+0$. The ket -supervector $\Psi=\left(S_{R}, S_{A}, \mu_{R}, \mu_{A}\right)^{\mathrm{T}}$ contains both commuting complex variables $S_{R / A}$ and Grassmann anti-commuting variables $\mu_{R / A}$. For convergence of the Gaussian functional integrals containing $e^{i S[\Psi]}$ the $b r a$-supervector $\bar{\Psi}$ is given by $\bar{\Psi}=\Psi^{\dagger} \Lambda$ where $\Lambda=\operatorname{diag}\{1,-1,1,-1\}$. The SUSY trick allows to do averaging over disorder prior to doing the functional integral over $\Psi$. Thus we obtain $\left\langle e^{i S[\Psi]}\right\rangle=e^{-F}$, where

$$
\begin{aligned}
F & =\frac{1}{2} \sum_{i, j} \mathbf{g}_{i j} \operatorname{Str}\left[\hat{Q}_{i} \hat{Q}_{j}\right]-\frac{i \tilde{\omega}}{2} \sum_{i} \operatorname{Str}\left[\Lambda \hat{Q}_{i}\right] \\
& -i E \sum_{i} \operatorname{Str}\left[\hat{Q}_{i}\right]+F_{k i n} .
\end{aligned}
$$

Here $F_{k i n}=i \sum_{i j} \bar{\Psi}_{i} H_{i j}^{(0)} \Psi_{j}$ and

$$
\hat{Q}=\Psi \otimes \bar{\Psi}=\Psi \otimes \Psi^{\dagger} \Lambda .
$$

So far we did not deviate from the standard rout except for introducing the new notations Eq.(2). However, the integration measure is still unchanged $d \mu[\Psi]=$ $d S_{R} d S_{A} d S_{R}^{*} d S_{A}^{*} d \mu_{R} d \mu_{A} d \mu_{R}^{*} d \mu_{A}^{*}$.

Now we do the crucial step. Instead of performing the Hubbard-Stratonovich transformation which decouples the quartic in $\Psi$ term in Eq. (11) by the supermatrix $Q$ and applying the subsequent saddle point approximation which leads to the sigma-model constraint $Q^{2}=1$ we change the variables so that in the new variables the supermatrix $\hat{Q}$ is parameterized similar to the supermatrix $Q$ of the sigma-model:

$$
\begin{aligned}
& S_{R / A}= \pm i \sqrt{\lambda_{1 / 2}} e^{ \pm i \varphi / 2+i \Omega}\left(1-\frac{1}{2} \chi_{R / A}^{*} \chi_{R / A}\right) \\
& \mu_{R / A}= \pm i \sqrt{\lambda_{1 / 2}} e^{ \pm i \varphi / 2+i \Omega} \chi_{R / A}
\end{aligned}
$$

where $\lambda_{1 / 2} \geq 0,0 \leq \varphi \leq 2 \pi, 0 \leq \Omega \leq \pi$ and $\chi_{R / A}, \chi_{R / A}^{*}$ are the new Grassmann variables.

One can check that in new variables the supermatrix $\hat{Q}$ defined in Eq.(2) can be represented in the form where Grassmann and commuting variables are separated by factorization:

$$
\hat{Q}=\left(\begin{array}{cc}
u_{R} & 0 \\
0 & u_{A}
\end{array}\right)\left(\begin{array}{ll}
\hat{D}_{R R} & \hat{D}_{R A} \\
\hat{D}_{A R} & \hat{D}_{A A}
\end{array}\right)\left(\begin{array}{cc}
u_{R}^{-1} & 0 \\
0 & u_{A}^{-1}
\end{array}\right) .
$$

The factorization Eq. (44) is also one of the basic properties of the Efetov's parameterization in the sigma-model [3]. Moreover, the corresponding matrices $u_{R / A}$ are essentially the same:

$$
u_{R / A}=\left(\begin{array}{cc}
1-\frac{1}{2} \chi_{R / A}^{*} \chi_{R / A} & -\chi_{R / A}^{*} \\
\chi_{R / A} & 1+\frac{1}{2} \chi_{R / A}^{*} \chi_{R / A}
\end{array}\right)_{B F} .
$$

The difference is in the form of the matrix $\hat{D}$. Like in the case of Efetov's parameterization it is diagonal in the boson-fermion (BF) space. However, in contrast to the sigma-model only the bosonic sector is nonzero:

$$
\hat{D}_{B B}=\left(\begin{array}{cc}
\lambda_{1} & \sqrt{\lambda_{1} \lambda_{2}} e^{i \varphi} \\
-\sqrt{\lambda_{1} \lambda_{2}} e^{-i \varphi} & -\lambda_{2}
\end{array}\right)_{R A}, \quad \hat{D}_{F F}=0 .
$$

One can see that the matrix $\hat{D}_{B B}$ can be diagonalized by the same pseudo-unitary transformation as the matrix $D_{B B}$ of the sigma-model: $\hat{D}_{B B}=\hat{T} \hat{B}_{0} \hat{T}^{-1}$, where $\hat{T} \in U(1,1)$. However, the diagonal matrix $\hat{B}_{0}=\left|\lambda_{1}-\lambda_{2}\right| \operatorname{diag}\left(\theta\left(\lambda_{1}-\lambda_{2}\right), \theta\left(\lambda_{2}-\lambda_{1}\right)\right)_{R A}$ is different from the corresponding diagonal matrix $\Lambda_{B B}$ of the sigma-model and contains a new degree of freedom, the difference $\lambda_{1}-\lambda_{2} \in \mathbb{R}$. This means that the full symmetry of the target space is $\frac{\mathbf{U}(\mathbf{1}, \mathbf{1})}{\mathbf{U}(\mathbf{1}) \times \mathbf{U}(\mathbf{1})} \times \mathbb{R}$ rather than $\frac{\mathbf{U}(\mathbf{1}, \mathbf{1})}{\mathbf{U}(\mathbf{1}) \times \mathbf{U}(\mathbf{1})} \times \frac{\mathbf{U}(\mathbf{2})}{\mathbf{U}(\mathbf{1}) \times \mathbf{U}(\mathbf{1})}$ as for the sigma-model.

To complete the procedure of changing variables from $\Psi$ to $\hat{Q}$ we have to compute the Jacobian of the transformation Eq.(3) and rewrite $F_{k i n}$ in Eq.(1) in terms of $\hat{Q}$. The first task is straightforward and the result is:

$$
d \mu \equiv d \hat{Q} d \Omega=d \chi_{R} d \chi_{R}^{*} d \chi_{A} d \chi_{A}^{*} d \varphi d \lambda_{1} d \lambda_{2} \frac{d \Omega}{\lambda_{1} \lambda_{2}} .
$$

To accomplish the second one we note that $\bar{\Psi}_{i}=\bar{\varphi}_{i} e^{-i \Omega_{i}}$ and $\Psi_{j}=\varphi_{j} e^{i \Omega_{j}}$ where $\varphi_{i}=\left.\Psi_{i}\right|_{\Omega_{i}=0}$. Then each link $\{i j\}$ enters in the last term of Eq.(11) as $i V_{i j}=$ $i\left(p_{i j} e^{-i \Omega_{i j}}+p_{j i} e^{i \Omega_{i j}}\right)$, where $p_{i j}=\bar{\varphi}_{i} \varphi_{j} H_{i j}^{(0)}$, and $\Omega_{i j}=$ $\Omega_{i}-\Omega_{j}$. Then assuming that the observable of interest is gauge-invariant and thus independent of $\Omega$ and integrating $e^{-i \sum_{i j} V_{i j}}$ over all $\Omega_{i j} \in[-\pi, \pi]$ one obtains for the last term in Eq.(1):

$$
F_{k i n}=-\sum_{j>i} \ln \left\{2 \pi J_{0}\left(2 \sqrt{\left|H_{i j}^{(0)}\right|^{2} \operatorname{Str}\left[\hat{Q}_{i} \hat{Q}_{j}\right]}\right)\right\} .
$$

Note, however, that in order to arrive at Eq.(8) integration over all $\Omega_{i j}$ should be independent. This is the case when the links provided by $H_{i j}^{(0)}$ form a lattice with no loops. The simplest example considered below is a one-dimensional lattice with only nearest neighbor connections $H_{i, i \pm 1}^{(0)}$.

The new supermatrix field theory given by Eqs.(11), (8) together with the parameterization of the supermatrix $\hat{Q}$, Eqs.(44)-(6) is the main result of the paper. It has to 
be compared with the standard supermatrix sigma-model [3]. In the case $\left\langle H_{i j}\right\rangle=0$ and $\mathbf{g}_{i j}=\mathbf{g}(|i-j|)$ the action reads [6]:

$F_{s m}=-\frac{\left(\pi \nu A_{0}\right)^{2}}{2} \sum_{i j} \mathbf{g}_{i j}^{-1} \operatorname{Str}\left[Q_{i} Q_{j}\right]-\frac{i \pi \nu \tilde{\omega}}{2} \sum_{i} \operatorname{Str}\left[\Lambda Q_{i}\right]$,

where $\nu(E)=\frac{1}{4 \pi A_{0}} \sqrt{8 A_{0}-E^{2}}$ is the mean density of states and $A_{0}=\sum_{i} \mathbf{g}_{i j}$. Comparing Eq. (9) with Eq.(1) one notices that the coupling matrix $\mathbf{g}$ in Eq.(1) is replaced in Eq.(9) by the inverse coupling matrix $\mathrm{g}^{-1}$. That is why the new representation is dual to the standard sigma-model representation. However the target spaces of the supermatrices $\hat{Q}$ and $Q$ in Eq.(1) and Eq.(9) are different. The $Q$-field is constrained: $Q^{2}=1$ and $\operatorname{Str} Q=0$. That is why the term $E \operatorname{Str} Q$ cannot arise and the energy $E$ enters as an inessential parameter. There is no such constrains for the supermatrix $\hat{Q}$ in Eq. (11) and the terms $E \operatorname{Str} \hat{Q}$ and $\mathbf{g}_{i i} \operatorname{Str} \hat{Q}_{i}^{2}$ are important. In particular this leads to peculiar properties of the $1 \mathrm{~d}$ disordered chain at the point $E=0$ 19, 20], especially in the chiral case 11] where diagonal matrix elements (on-site energies) $H_{i i}$ do not fluctuate.

1d Anderson model.-We illustrate the new method by considering the statistics of an eigenfunction $\psi_{n}(i)$ corresponding to the eigenvalue $E_{n}$ for the 1d Anderson model. This problem has been studied earlier [18] by a different method which was not based on the SUSY trick. The 1d Anderson model corresponds to the field theory Eqs.(1), (8) where the coupling matrix $\mathbf{g}_{i j}=w \delta_{i j}$ is diagonal and $H_{i j}^{(0)}=\delta_{i, j+1}+\delta_{i, j-1}$. To compute the distribution function $P_{i}(t)=\nu^{-1}(E)\left\langle\sum_{n} \delta\left(t-\left|\psi_{n}(i)\right|^{2}\right) \delta\left(E_{n}-\right.\right.$ $E)\rangle$ we follow the procedure used in [3, 5] for thick quasi$1 \mathrm{~d}$ wires where the sigma-model applies. It starts by the exact expression for the $q$-th moment of this distribution

$$
I_{q}(i)=\frac{1}{4 \pi \nu(E)} \frac{1}{(q-2) !} \int_{0}^{\infty} d s s^{q-2} \int_{-\infty}^{+\infty} d v Y_{i}(s, v)
$$

in terms of the function $Y\left(\hat{Q}_{i}\right)=\int \prod_{\ell \neq i} d \hat{Q}_{\ell} e^{-F(\hat{Q})}$. The functional $F$ is given by Eq.(11), where $\tilde{\omega}=$ $2 \eta \rightarrow+0$ and $F_{k i n}=\sum_{i} F_{k i n}\left(\hat{Q}_{i}, \hat{Q}_{i+1}\right)=$ $-\sum_{i} \ln 2 \pi J_{0}\left(2 \sqrt{\operatorname{Str} \hat{Q}_{i} \hat{Q}_{i+1}}\right)$.

As usual [5] in the 1d chain of the length $N$ the function $Y_{i}=W_{i} W_{N-i} e^{-L\left(\hat{Q}_{i}\right)}$ is found from the recursive relation:

$$
W_{j+1}(\hat{Q})=\int d \mu\left(\hat{Q}^{\prime}\right) e^{-F_{k i n}\left(\hat{Q}, \hat{Q}^{\prime}\right)-L\left(\hat{Q}^{\prime}\right)} W_{j}\left(\hat{Q}^{\prime}\right),
$$

where $L(\hat{Q})=\frac{w}{2} \operatorname{Str} \hat{Q}^{2}+\operatorname{Str} \Lambda \hat{Q}-i E \operatorname{Str} \hat{Q}$, and the boundary condition $W_{j=1}(\hat{Q})=1$ is assumed.

One can show that the integral in Eq. (11) does not depend on the Grassmann variables $\chi$ and on the angle $\varphi$. Thus $W_{i}$ and $Y_{i}$ are functions of two $\left(\lambda_{1}, \lambda_{2}\right)$ of the seven variables only. This property is based on the form of the parameterization Eqs.(4)-(6) and similar to that known in the sigma-model approach [3, 5, 21], where the corresponding functions depend on the non-compact $\lambda_{B}$ and the compact $\lambda_{F}$ angles only. In the limit $\eta \rightarrow+0$, $W_{i}$ remains a function of two new variables $v=\lambda_{1}-\lambda_{2}$ and $s=\eta\left(\lambda_{1}+\lambda_{2}\right)$. At this point the difference in the symmetries of the target spaces in our approach and the sigma-model becomes important. In the latter case $W_{i}$ depends on one rescaled variable $(2 \eta) \lambda_{B}$ only, while the dependence on $\lambda_{F}$ disappears [3, 5, 21]. The fact that the recursive relation Eq.(11) and the Fokker-Plank equation that follows from it depend on two variables is generic to all methods in the theory of $1 \mathrm{~d}$ disordered chains 22] thus demonstrating that the SUSY approach is the minimal description. We will see that the dependence on $v$ reflects statistics of eigenfunction oscillations at scales of the order of the de Broglie wavelength $k_{F}^{-1}$ which are essentially different in a thick wire and in a $1 \mathrm{~d}$ chain.

After integration over the angle $\varphi^{\prime}$ and over the Grassmann variables according to a convention $\int \chi_{R / A}^{\prime} d \chi_{R / A}^{\prime}=\frac{1}{\sqrt{2 \pi}}$ and going to the limit $\eta \rightarrow 0$ one obtains an exact recursive equation:

$$
\begin{aligned}
W_{j+1}(s, v) & =\frac{s^{\frac{1}{2}}}{2 \pi} \int_{\infty}^{+\infty} d v^{\prime} \int_{0}^{\infty} \frac{d s^{\prime}}{\left(s^{\prime}\right)^{\frac{3}{2}}} W_{j}\left(s^{\prime}, v^{\prime}\right) \\
& \times \cos \left[\sqrt{s s^{\prime}}\left(\frac{v^{\prime}}{s^{\prime}}+\frac{v}{s}\right)\right] e^{-s^{\prime}-\frac{w}{2} v^{\prime 2}+i E v^{\prime}}
\end{aligned}
$$

Similar equations have been derived earlier [23, 24] in study of the localization transition on the Bethe lattice. Eq. (12) is valid for any strength of disorder $w$ and for an arbitrary energy $E$. However in the limit of weak disorder $w \ll 1$ it can be drastically simplified using the scale separation $k_{F}^{-1} \ll \xi_{\text {loc }}$ where $\xi_{\text {loc }}$ is the localization length. To this end we first consider the disorderfree case. Setting $w=0$ in Eq. (12) one notices that the Fourier-transform $\tilde{W}_{j}\left(s^{\prime}, q^{\prime}\right)=\int d v^{\prime} W_{j}\left(s^{\prime}, v^{\prime}\right) e^{i v^{\prime} q^{\prime}}$ naturally arises. Remarkably, the equation for $\tilde{W}_{j}(s, q)$ turns out to be an algebraic and not an integral equation: $\tilde{W}_{j+1}(s, q)=\frac{1}{q^{2}} e^{-s q^{2}} \tilde{W}_{j}\left(s q^{2}, E-\frac{1}{q}\right)$. This functional equation can be simplified if we introduce new variables $s=z \cos ^{2}(\phi / 2+k)$ and $q=\cos (\phi / 2) / \cos (\phi / 2+k)$ and a new function:

$$
\Phi_{j}(z, \phi)=\tilde{W}_{j}(s(z, \phi), q(z, \phi)) \frac{\sin k}{2 \cos ^{2}(\phi / 2+k)},
$$

where $k$ defined by the relation $E=2 \cos k$ is a momentum for plane waves in the tight-binding model.

In new variables we obtain:

$$
\Phi_{j+1}(z, \phi)=e^{-z \cos ^{2}(\phi / 2)} \Phi_{j}(z, \phi-2 k) .
$$

One can see that the variable $\phi / 2$ is related with the phase of the wave function as it changes by $k$ in passing 
from a lattice site $j$ to the neighboring one. For an irrational $k / \pi$ as one moves along the chain at a distance $\xi_{l o c} \gg 1$, the phase $\phi$ sweeps with the constant density through the entire interval $[0,2 \pi]$. Therefore $\phi$ is the true "fast" variable 22] in contrast to the "slow" variable $z$. Thus one comes to an idea 22] of averaging over $\phi$ and replacing $\Phi_{j}(z, \phi)$ in Eq. (13) by its average (the "fast phase" ansatz): $\Phi_{j}(z) \equiv \overline{\Phi_{j}(z, \phi)}=\frac{1}{2 \pi} \int_{0}^{2 \pi} d \phi \Phi_{j}(z, \phi)$.

Now we take into account weak disorder by expanding $e^{-\frac{w}{2} v^{\prime 2}}=1-\frac{w}{2} v^{\prime 2}=1+\frac{w}{2} \partial^{2} / \partial q^{\prime 2}$ in Eq. (12). We also assume $z \sim w \ll 1$ and expand $e^{-z \cos ^{2}(\phi / 2)} \approx$ $1-z \cos ^{2}(\phi / 2)$. Then doing the Fourier transform over $v$ and $v^{\prime}$, switching to the new variables $z, \phi$ and averaging over $\phi$ one obtains for $\Phi_{j+1}(z)-\Phi_{j}(z)=$ $c_{1} z \Phi_{j}(z)+c_{2} z^{2} \frac{\partial^{2} \Phi_{j}}{\partial z^{2}}+c_{3}\left(\Phi_{j}-z \frac{\partial \Phi_{j}}{\partial z}\right)$, where $c_{1}=$ $-\overline{\cos ^{2}(\phi / 2)}=-1 / 2, c_{2}=\frac{w}{\sin ^{2} k} \overline{\left(1-\cos ^{2} \phi\right)}=\frac{w}{2 \sin ^{2} k}$, and $c_{3}=\frac{w}{\sin ^{2} k} \overline{(1+\cos \phi)(1-2 \cos \phi)}=0$. Then switching to the continuous spacial variable $\tau=j / \xi_{\text {loc }}$ with $\xi_{l o c}=\frac{2 \sin ^{2} k}{w}$ and the variable $x=z \xi_{l o c} / 2$ we obtain the following Fokker-Plank equation subject to the boundary condition $\Phi_{\tau=0}(x)=1$ :

$$
\frac{\partial \Phi_{\tau}(x)}{\partial \tau}=\left(x^{2} \frac{\partial^{2}}{\partial x^{2}}-x\right) \Phi_{\tau}(x) .
$$

The fact that $c_{3}=0$ depends crucially on the assumption on the homogeneous distribution of the fast phase $\phi$ which is related with the single-parameter scaling. As a matter of fact the distribution of phases $F(\phi ; E / w)$ depends on energy and is homogeneous $F(\phi ; \infty)=\frac{1}{2 \pi}$ only for irrational $k$ away from the band center $E=0$. At the band center $F(\phi ; 0) \propto(3+\cos \phi)^{-1 / 2}$ is different 25] and we obtain $c_{1}=1-2 E / K \approx-0.457$, $c_{2} \frac{\sin ^{2} k}{w}=(-16 / 3+8 E / K) \approx 0.494$ and $c_{3} \frac{\sin ^{2} k}{w}=$ $(-26 / 3+12 E / K) \approx 0.075$, where $E=E(1 / 2)$ and $K=K(1 / 2)$ are elliptic integrals. Thus at the band center $|E| \ll w$ Eq. (15) should be modified by including the combination $\Phi-x \partial \Phi / \partial x$ proportional to $c_{3}$.

Finally we have to switch to new variables in Eq. (10) which involves the combination $\tilde{W}_{i}(s, q) \tilde{W}_{N-i}(s, E-q)$. After replacing $\tilde{W}$ by $\Phi$ according to Eq. (13), using the fast phase ansatz $\Phi_{j}(z, \phi) \rightarrow \Phi_{\tau}(x)$ and integrating over $\phi$ one obtains for $|E| \gg w$ :

$$
I_{q}(\tau)=\frac{(2 q-1) ! !}{(q !)^{2}} \int_{0}^{\infty} d y q(q-1) y^{q-2} Y_{\tau}\left(\xi_{l o c} y\right),
$$

where we used the relation $2 \pi \nu(E)=d k / d E=$ $1 /(2 \sin k)$ and the definition $Y_{\tau}(x)=\Phi_{\tau}(x) \Phi_{\frac{N}{\xi_{l o c}}-\tau}(x)$. The result given by Eqs.(15),(16) is in a perfect agreement with the one obtained in Ref. 18 by a completely different method.

Remarkably, the integral in Eq.(16) and the FokkerPlank equation Eq. (15) coincide completely [5] with the corresponding equations [3, 5] determining statistics of wave functions for a multi-channel disordered wire. The $q$-dependent factor in front of the integral in Eq. (16) is due to the difference in statistics of oscillations of almost ballistic (in a strictly $1 \mathrm{~d}$ system) and fully chaotic (in a thick wire) wave functions at a small scale of the order of the de Broglie wavelength. This coincidence is a manifestation of the single-parameter scaling and it breaks down near the band center $|E| \ll w$.

* Electronic address: aossipov@ictp.trieste.it

[1] F. Wegner, Z. Phys 35, 207 (1979).

[2] K. B. Efetov, Adv. Phys. 32,53 (1983).

[3] K. B. Efetov, Supersymmetry in Chaos and Disorder, (Cambridge University Press, 1997).

[4] L. P. Gorkov, A. I. Larkin, and D. E. Khmelnitskii, Pis'ma v Zh. Eksp. Teor. Fiz. 30, 248 (1979) [JETP Letters 30228 (1979)].

[5] A. D. Mirlin, Phys. Rep. 326, 259 (2000).

[6] Y. V. Fyodorov and A. D. Mirlin, Phys. Rev. Lett. 67, 2405 (1991).

[7] V. L. Berezinskii, Zh. Eksp. Teor. Fiz. 65, 1251 (1973) [Sov. Phys. JETP 38, 620 (1974)].

[8] A. A. Abrikosov and I. A. Ryzhkin, Adv. Phys. 27, 147 (1978).

[9] V. I. Melnikov, JETP Letters 32, 225 (1980).

[10] N. F. Mott, Adv. Phys. 16, 49 (1967).

[11] F. J. Dyson, Phys. Rev. 92, 1331 (1958).

[12] M. R. Zirnbauer, J. Math. Phys. 37, 4986 (1996); A. Altland and M. R. Zirnbauer, Phys. Rev. B 55, 1142 (1997).

[13] O. M. Yevtushenko and V. E. Kravtsov, J. Phys. A 36, 8265 (2003)

[14] A. D. Mirlin et al. Phys. Rev. E 54, 3221 (1996).

[15] V. E. Kravtsov and K. A. Muttalib, Phys. Rev. Lett. 79, 1913 (1997).

[16] K. B. Efetov, G. Schwiete, and K. Takahashi, Phys. Rev. Lett. 92, 026807 (2004).

[17] J. Polchinski, String Theory, v.I, (Cambridge University Press, 1998), p.231.

[18] I. V. Kolokolov, Zh. Eksp. Teor. Fiz. 103, 2196 (1993) [Sov. Phys. JETP 76, 1099 (1993)].

[19] H. Schomerus and M. Titov, Phys. Rev. B 67, 100201(R) (2003).

[20] L. I. Deych, M. V. Erementchouk, A. A. Lisyansky, and B. L. Altshuler, Phys. Rev. Lett. 91, 096601 (2003).

[21] M. R. Zirnbauer, Nucl. Phys. B 265, 375 (1986).

[22] I. M. Lifshits, S. A. Gredeskul, L .A. Pastur, Introduction to the theory of disorederd systems, John Wiley and Sons, New York, 1988, p.147.

[23] A. D. Mirlin and Y. V. Fyodorov, Nucl. Phys. B 366, 507 (1991).

[24] R. Abou-Chacra, P. W. Anderson, and D. J. Thouless, J. Phys. C 6, 1734 (1973)

[25] A. Ossipov, T. Kottos, and T. Geisel, Phys. Rev. B 61, 11411 (2000). 